\title{
Sex and Menstrual Phase Influences on Sleep and Memory
}

\author{
Alejandra Alonso ${ }^{1}$ (D) $\cdot$ Lisa Genzel ${ }^{1} \cdot$ Angela Gomez $^{2}$
}

Accepted: 28 December 2020 / Published online: 10 March 2021

(C) The Author(s) 2021

\begin{abstract}
Purposes of Review This review highlights the effect of sex differences in sleep mediated memory consolidation and cognitive performance. In addition, the role of menstrual cycle and the fluctuating level of sexual hormones (mainly oestrogen and progesterone) are stressed.

Recent Findings The literature indicates that sex hormones mediate and orchestrate the differences observed in performance of females in comparison with males in a variety of tasks and can also be related to how sleep benefits cognition. Although the exact mechanism of such influence is not clear, it most likely involves differential activation of brain areas, sensitivity to neuromodulators (mainly oestrogen), circadian regulation of sleep and temperature, as well as modification of strategies to solve tasks across the menstrual cycle.

Summary With the evidence presented here, we hope to encourage researchers to develop appropriate paradigms to study the complex relationship between menstrual cycle, sleep (its regulation, architecture and electrophysiological hallmarks) and performance in memory and other cognitive tasks.
\end{abstract}

Keywords Menstrual cycle $\cdot$ Humans $\cdot$ Rodents $\cdot$ Sex differences $\cdot$ Cognition $\cdot$ Memory $\cdot$ Sleep $\cdot$ Sleep-regulation

\section{Introduction}

Sex is a variable known to influence diverse aspects of brain and behaviour, but surprisingly, sex differences and their consequences are often overlooked and are considered negligible by many researchers. Instead, rodent and human studies regard male and female subjects as simply equal and opt to include only males; thus, females end up being underrepresented in neuroscience research [1]. In this review, in addition to general sex differences, we also discuss how a specific aspect of sex differences (the menstrual cycle) may exert influences in sleep and learning in the context of sleep's contribution to cognition. We start with the general effects on sleep, continue with effects on cognition and memory and finish

This article is part of the Topical Collection on Sleep and Learning

Alejandra Alonso

a.alonsogarrido@donders.ru.nl

1 Department of Neuroinformatics, Faculty of Science, Donders Institute for Brain, Cognition and Behaviour, Radboud University, P.O. Box 9010, 6500 GL Nijmegen, The Netherlands

2 Department of Psychology, Universidad de los Andes, Cra. $1 \mathrm{~N}^{\circ}$ 18A-12, Bogota, Colombia with studies that include sleep, learning and memory. Within each section, we describe sex driven differences as well as menstrual phase differences, focusing in common results across human and rodent studies. While rodent studies present with the opportunity for controlled designs (i.e. administration of hormones and gonadectomization) and limiting the influence of confounders such as self-reported sleep and cultural bias, they rely on behavioural measures as proxy of the subjacent cognitive skill, requiring extra efforts to clarify a particular strategy taken by the individual to solve an experimental task. Human studies are often weak in control and can only capture snapshots of the desired variable studied, which can be an important limitation in a phenomenon of repetitive nature such as menstrual cycle. Both types of studies offer a window into the fluctuating influence of menstrual cycle and sex hormones in the functioning of female individuals.

\section{Influence of Sex on Sleep}

Sex differences can be anatomical or functional [2] and express themselves both at volumetric and connective levels [3]. Those differences emerge early on during development and are later reinforced by exposure to sex steroids $[4 \bullet \bullet]$. 
Circulating gonadal hormones exert their effects on not only secondary sexual features but also most brain regions express oestrogen and progesterone receptors. These brain regions include sleep regulating areas such as the ventrolateral preoptic $[2,5]$ median preoptic and suprachiasmatic nuclei, lateral hypothalamus [3], as well as areas involved in cognition and memory such as the prefrontal cortex [6] and the hippocampal formation $[7,8]$.

Sleep presents itself in a complex form, featuring behavioural (for instance, diminished response to stimuli), as well as physiological changes compared with wakefulness (such as brain activity that displays distinct patterns of neuronal firing). In most animals, it is possible to differentiate between two main sleep stages: non-rapid eye movement sleep (NREM) and rapid eye movement sleep (REM) [9]. NREM is distinguishable by its higher amplitude, slower waves relative to wake, while REM displays a more similar brain activity pattern to wakefulness, with low amplitude and high frequency waves [10] (Fig. 1a). Muscle atonia and the characteristic eye movements (similar to saccades) that give name to the state are also a hallmark of REM.

\section{Rodents}

When studying sleep in rodents, it is important to note that, contrary to humans, they are most active during the dark phase, and their sleep pattern is polyphasic, with several bouts of sleep during both light and dark phases of the day (see Fig. $1 b)$.

Studies on spontaneous sleep show that female mice have less total sleep and daily NREM compared with males [11-13] and, during the dark phase, males exhibit more wake-sleep shifts, while females have longer bouts of wakefulness [12]. REM is also decreased in female rats [13] and mice [11]. Gonadectomy in mice of both sexes eliminates the differences seen in total spontaneous sleep and NREM, as well as in delta power and sleep fragmentation, which implies that the differences in current hormones levels are responsible for these variations in sleep and not general developmental differences. Ovariectomized rats show decreased REM and total sleep time during the light phase, compared with castrated males [14]. When treated with stable levels of $17 \beta$ oestradiol or oestradiol and progesterone, they show increases in wake and decreases in REM and NREM during the dark phase [15-17].

Studies on recovery sleep after sleep deprivation in mice show for both sexes decreased NREM the first $2 \mathrm{~h}$ of recovery sleep, followed by an increase during the first half of the dark phase, but this rebound is greater in females [13]. Female rats also showed an increased NREM rebound during recovery sleep as well as increased delta power compared with males $[18 \bullet]$. Studies on gonadectomized rats with hormone implants show that during recovery sleep oestradiol and progesterone promote REM during the light phase and reduces NREM delta power $(1-4 \mathrm{~Hz})$ during the dark phase $[16,19]$. Gonadectomy on mice of both sexes eliminates most of the differences seen in recovery sleep after sleep deprivation [13].

To further study the effect of sex chromosomes in sleep, the core four-genotype mouse model [20] allows to have females and males with either XX or XY chromosomes. This is a transgenic line that manipulates the presence or absence of the Sry gene, which determines testes development and is normally found on the Y chromosome. By knocking Sry out of the Y chromosome, they can obtain females XY, and by knocking Sry into the X chromosome, they can obtain males XX. Studies in this line showed that genetic sex does not influence sex differences in spontaneous sleep, but rather has an impact in recovery sleep, where XY females had more total recovery sleep and more NREM than XX females at the midpoint of the dark phase, suggesting that increased sleep propensity is linked to the $\mathrm{Y}$ chromosome. In gonadectomized animals, after sleep deprivation, the dissipation of delta power was quicker in XX males than XX females [12], indicating that in the absence of female gonadal hormones, the advantage that females had in recovery sleep compared with males is lost.

\section{Humans}

Overall, female humans display more consolidated sleep (less awakenings), shorter sleep latencies and lower percentage of light sleep, than aged-matched males [3,21], and this is consistent for different age groups and experimental assessments of sleep (laboratory vs home sleep-recoding conditions). Paradoxically, women report consistently worse subjective sleep quality than men. Females tend to describe their sleep quality as poor due to nighttime disruptions, insufficient quantity and long sleep latencies. Women also claim suffering from insomnia in a greater proportion than men [22, 23], and this disruption grows stronger as women age (with menopause onset) [24].

Concerning circadian regulation of sleep, women seem to have shorter and earlier rhythms of temperature change and melatonin secretion (induces drowsiness and sleep) under a normal schedule [3]. It has also been documented that women go to bed and wake up earlier than men [25] Since often, women extend the time before going to sleep to after the melatonin peak, this could be related to their complain of longer sleep onset latencies [23] due to the missing of the optimal timewindow for sleep onset. However, under a 28-h desynchrony paradigm ( $9.5 \mathrm{~h}$ sleep and $18.5 \mathrm{~h}$ wake), women show lower accuracy and exert more effort in cognitive tests after prolonged wakefulness compared with men [26].

In terms of the features of brain oscillatory events during sleep (i.e. power of electroencephalography signal and its frequency), Carrier and colleagues [27] showed, in a sample of 
a

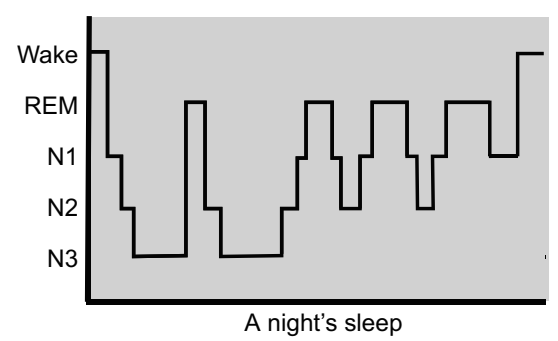

C Estrous cycle

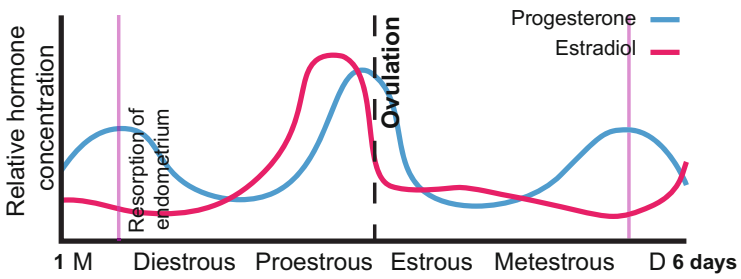

Fig. 1 Diagrams of the menstrual cycle and sleep patterns in humans and rodents. a Sleep pattern in humans. N1 is the transition state into sleep, characterized by drowsiness and a low arousal threshold. N2 is a deeper sleep than N1, during which breathing and heartbeat decrease. It is also in this state when sleep spindles can be observed. N3 also known as slow wave sleep is characterized by a predominance of delta oscillations, with the EEG signal showing slow frequencies and high amplitude waveforms, believed to be a regenerative period during sleep. REM sleep or paradoxical sleep is characterized by an EEG state of high frequency and low amplitude oscillations, similar to wake, but with complete muscle atonia. The line depicts transitions between states throughout the night, with REM episodes becoming longer as the night progresses. b Sleep patterns in rodents. These animals sleep the most during the light cycle, where they have numerous short bouts of sleep

men and women between 20 and 60 years, that women display higher power in diverse physiological frequencies considered during sleep (delta $0.1-4 \mathrm{~Hz}$, theta $4-7 \mathrm{~Hz}$ and alpha $8-12 \mathrm{~Hz}$ and spindles $14-16 \mathrm{~Hz}$ ). Spindles are a prominent feature of NREM sleep and have been associated, with intelligence and learning capabilities $[28,29]$. A difference in spindle power between the sexes has been consistently described [30] and may be related to the variations in spindle topography [31]. For instance, a study by Huupponen and colleagues [32] found that women had a higher percentage of spindles in the left frontal electrode (Fp1-A2) and men displayed more spindles in the occipital electrodes (O2-A1). However, it is not completely clear whether this is due to lower skull thickness in women, since skull thickness could influence surface electroencephalography power and thus spindle detection [33••].

Regarding slow wave activity (SWA), a low-frequency (2$4 \mathrm{~Hz}$ ) activity observed in NREM and a proxy for sleep pressure, men and women do not show differences in power, but the proportion of SWA was more strongly regulated by circadian rhythms in women than in men. As for age effects on sleep, mid-age women display greater percentage of SWA than same-age men [27] and SWA/delta decline is less severe in women than in men $[24,25]$. In the same direction, women b

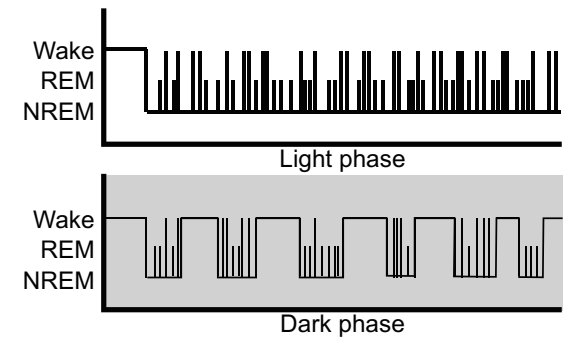

\section{d Menstrual cycle}

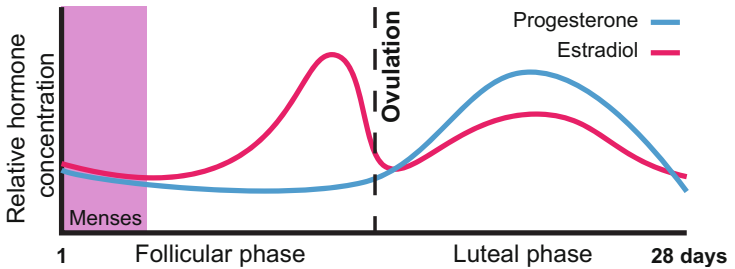

and wake throughout the day. During their active phase, they also sleep, with bouts of short sleep-wake transitions followed by a wake period, back to the sleep bouts. $\mathbf{c}$ Oestrous cycle in rodents, divided in diestrous, proestrous, oestrous and met oestrous for an average of 5 days. During proestrous, a peak of estradiol followed by a peak of progesterone gives place to ovulation. Rodents do not menstruate; instead, they experience a resorption of the endometrium, marked as magenta lines. d Menstrual cycle in humans is divided into follicular phase and luteal phase, with an average length an average of 28 days. On the last days of the follicular phase, estradiol increases, followed by a spike of luteinizing hormone which drives ovulation. The corpus luteum remaining secretes progesterone, and when both progesterone and estradiol are low, the uterine lining detaches along with the unfertilized egg, with the menses marked as a magenta box

display greater SWA rebound after sleep deprivation, which has been interpreted as women accumulating a greater sleep debt than men and making them more sensitive to sleep loss due to work-shifts and more propense to accidents [4••].

\section{Interim Summary}

In sum, across both rodents and humans, sleep differs between the sexes for variables such as sleep architecture and its circadian regulation, as well as features of electrophysiological events characteristic of sleep (for instance, spindles and slow wave activity differ in their power and frequency). However, in terms of observed sleep quality, the direction of the difference seems to be opposite among rodent findings and human studies, since the latter point towards better sleep- quality in females not seen in female rodent models.

\section{Influences of Menstrual Cycle on Sleep}

After puberty, females experience on a regular basis changes in the reproductive system that allow for pregnancy to occur, known as the menstrual cycle. It allows oocytes to mature and 
prepares the uterus by thickening the uterine lining. Different hormones produced by the hypothalamus and the gonads orchestrate the different stages of the menstrual cycle; however, these hormones affect not only the reproductive system but also the nervous system. In this section, we will highlight how the menstrual cycle influences sleep on both humans and rodents.

\section{Rodents}

In rodents, the oestrous cycle lasts around 5 days and is divided in proestrous, oestrous, metestrous and diestrous. Proestrous occurs the day before ovulation, with the highest concentration of progesterone and oestradiol, the highest peak is at the end of the light phase for oestradiol, and at the start of the dark phase for progesterone. Oestrous is ovulation day, with oestradiol and progesterone starting to decay; during metestrous and diestrous, oestradiol starts to increase gradually, with a small increase in progesterone. In most mammals, after ovulation, there is a luteal phase; however, in rodents, only if the female has been involved in sexual behaviours does the corpus luteum secretes progesterone [34] (see Fig. 1c).

Mice show little change in sleep distribution during the oestrous cycle, with variations between different mouse strains, but overall limited differences in oestrous cycle. For instance, in the C57 strain, there is decreased REM during the night of proestrous compared with diestrous [35], followed by an increase the next day [18 ${ }^{\bullet}$; this phenomenon is also seen in Sprague-Dawley rats [14]. On proestrous night, rats show an increase in wake and decrease in NREM compared with oestrous [26]. As for sleep architecture, proestrous females had a higher number of short duration wake bouts compared with oestrous, metestrous, and diestrous [18•]. These differences are dependent on light/dark cycles. During the light phase, REM sleep is not affected by the oestrous cycle, but in dark phase, REM is inhibited during proestrous and oestrous [14]; this has also been shown in ovariectomized rats with oestradiol replacement (which tries to mimic proestrous night) having decreased REM and NREM only during the dark phase [19].

\section{Humans}

In women, menstrual cycle can vary from 24 to 35 days, with an average of 28 days and is divided in (i) a follicular phase, during which the ovarian follicles develop and mature, and blood shedding occurs, (ii) an ovulation phase in which the mature egg is released and leaves the fallopian tube and (iii) a luteal phase characterized by the formation of the luteal corpus (uterine lining) [33*0]. On the first day of menses, the hormones regulating the cycle are low (progesterone, oestrogen luteinizing hormone, follicle-stimulating hormone). In contrast, during the luteal phase, follicle stimulating hormone and oestrogen rise; as the non-fertilized ovule degenerates, the production of hormones drops again [36]. Sexual steroid hormones levels and the feedback for their cyclical regulation depend on the central nervous system. The nature of the release of such substances is recurrent and pulsates across the cycle, and therefore, their effects are transient in nature [37] (Fig. 1d).

Sex differences in reported sleep quality begin in the adolescence, with the onset of sexual maturation (menarche in women) [23]. As previously mentioned, subjective sleep quality is lower in women but appears as specially affected before the menses [38] and women (mainly those affected by premenstrual disruptive symptom [36]) report insufficient and fragmented sleep [30]. Yet insomnia reports from women extend across all phases of the menstrual cycle [23], but insomnia worsens during the premenstrual phase (mid-luteal phase) and is linked to women's anxiety and perception of life-disruption due to the menses [3,23]. Paradoxically, oral contraceptives do not seem to influence subjective sleep quality [6].

Nonetheless, the International Classification of Sleep Disorders (ICSD-10) includes a category of menstrual sleep related disturbances: menstrual-related hypersomnia [2], which is described as consistent episodes of hypersomnia that begin 1 week before menses. In line with this, subjective daytime sleepiness was rated higher in the luteal phase by a group of women who were freely allowed to take 10 min naps in a 24-h day period. Slow wave sleep (a key component of NREM) was also more frequent during luteal phase compared with the follicular phase. Both hypersomnia and insomnia seem to be linked to abnormal temperature variation due to higher levels of progesterone and oestrogens [6].

Body temperature is also highly dependent of the hormone level and menstrual phase. Men and women are most similar in the circadian regulation of the body temperature in the follicular phase of the latter [38]. Body temperature in women seems to be at its lowest during the periovulatory days, due to the high level of oestradiol. In the luteal phase, body temperature increases due to high levels of progesterone. During the night, temperature falls, but during the luteal phase, night temperature shows a reduced amplitude decay, which has been attributed to the progesterone counteracting the hypothermic action of melatonin [6]. Wright and colleagues [39] studied the relationship between phase of the menstrual cycle, use of oral contraceptives and circadian regulation on alertness and body temperature in a constant routine paradigm. They did not find an effect of oral contraceptives or phase of the menstrual cycle in circadian phase, however during the luteal phase women showed higher levels of alertness (measure with the Psychomotor Vigilance Test) and this is related to lower daytime temperature. Melatonin levels were similar in luteal phase and follicular phase, but higher along night hours for women using oral contraceptives, who also displayed higher temperatures. 
Sleep continuity and sleep efficiency measured through polysomnographic recordings of healthy women remain stable across the different phases of the menstrual cycle [30]. Individual percentages of sleep stages are also comparable across phases. Only a shorter sleep latency and decrease percentage of REM sleep during the luteal phase are documented, but this effect appears only on the first sleep cycle [36]. These changes seem to be linked to the mentioned raise in body temperature that takes place during this phase [40]. As for the women using hormonal contraceptives, they have been associated with lower percentage of slow-wave sleep (SWS), shorter REM sleep onset latency and increased REM sleep efficiency in the active phase of the contraceptive method and this compared with women [3]. SWA and SWS do not appear to be sensitive to the influence of menstrual cycle; however, a transient increase during the first sleep-cycle was evident in a study by Driver and colleagues, and it was attributed to temperature increase during the luteal phase [40]. Spindle activity is reported as higher in women during their luteal phase [41], concretely their duration, number and spectral frequency increases during mid-luteal phase, especially after the first cycle of sleep [40]. The upper spindle frequency (14 to $16 \mathrm{~Hz}$ ) appears as particularly sensitive to menstrual fluctuation. Ishizuka and colleagues [42] report spindle frequency reaching a high peak 3 days before menses and a valley 18 days before the period. Plante and Goldstein [43] propose that this luteal enhancement of spindles can be attributed to higher levels of progesterone, as their sample of women taking the progestin drug Medroxyprogesterone acetate, displayed increased spindle density, power and amplitude in the spindle band $(11-16 \mathrm{~Hz})$. In this study, menstrual phase was not controlled. This effect was explained as progesterone being responsible for an increased binding in the reticular thalamic nucleus to GABA receptors, which may enhance spindles role in brain inhibition $[4,36]$.

\section{Interim Summary}

Regarding effects of menstrual cycle in sleep, findings are weaker than expected in both humans and rodents. In rodents, changes seem to be more related to sex than to hormonal variation with females spending more time in wake and less in NREM during the light phase. One exception is the observed decrease of REM on proestrous night and rebound of REM on the following oestrous day in rodents. In humans, effects of menstrual cycle in sleep are intertwined with body temperature changes across menstrual phases. The intersection of human and animal studies indicates that progesterone reduces arousal, while oestrogen increases the availability of norepinephrine, decreasing the time in REM sleep [6]. These findings are consistent with the documented anxiolytic/ sedative effect of oestrogen and progesterone in animal models $[6,44]$ and the reported decrease in sleep quality during the luteal phase and the frequent sleep disturbances displayed during menopause (due to the sudden drop of progesterone) [23].

Sleep's role in cognition and specially the consequences of its deprivation have been extensively studied in literature. Sleep is known to influence cognitive abilities [10, 45], including working memory, planning and, hence, it is expected that sleep differences in both sex and menstrual cycle will also affect learning and memory.

\section{Effects of Sex on Cognition}

As mentioned before, sex differences seem to permeate the organization of brain $[8,46]$ and even the mechanisms behind the observed cognitive performance in certain tasks, such as synaptic plasticity (in the long- and short-term) and activation of other molecular pathways (neurotransmitter's release, gene expression and transcriptional factors) [47]. It is difficult to distinguish between the cause of such differences, since sex hormones, sex chromosomes and environmental factors interact to enable cognition and behaviour. In this section, the most relevant findings regarding the differential performance of sex, in both humans and rodents in cognitive performance, are described.

\section{Rodents}

Studies of gonadal hormones and sex differences in learning have shown mixed results, probably because sex studies do not usually discern between the different stages of the oestrous cycle in females. Differences in hormone levels can alter the strategies used by rodents to perform different tasks. These differences in cognitive strategy seem to emerge after puberty in rats [48], one potential reason why the role of sexual hormones seems to be central. In general, as with humans, male rodents are attributed to have better spatial learning, which is expressed in better spatial working and reference memory [49, 50]. In the skilled reaching task (Fig. 2a), in which rats have to reach with their paws through a small opening for a food pellet, females had better performance during acquisition of the task compared with males. In a discriminative fearconditioned to context task (Fig. 2b), in which the rat has to associate a context with a foot shock, Long Evan females showed longer freezing time than males, showing an increased emotional memory to a particular context [53].

There are many factors to consider, apart from sex, when trying to compare these different findings, such as age, strain, and variations in the tasks themselves [54], as well as the housing conditions (enriched environments, physical activity, handling). At the same time, when studying the menstrual phase influences, studies introduce a large variation in timing, route, length and dosage of hormone administration. 


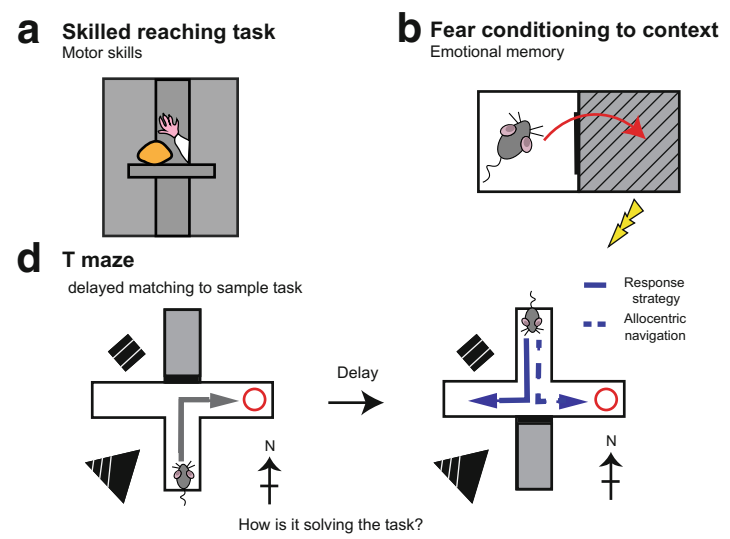

C Image rotation Which object corresponds to the original figure?
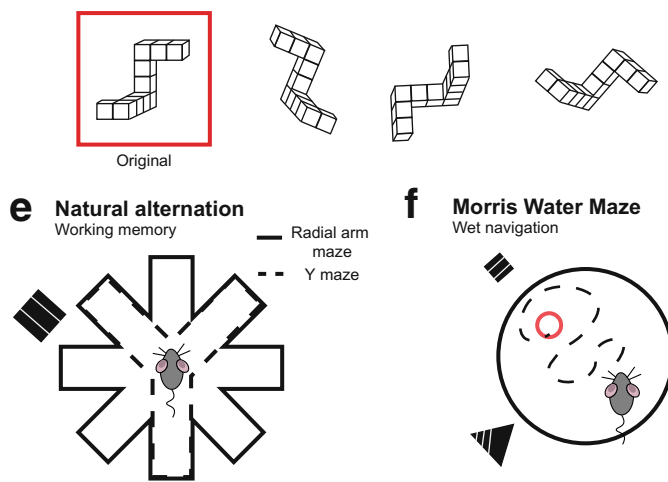

f Morris Water Maze Wet navigation
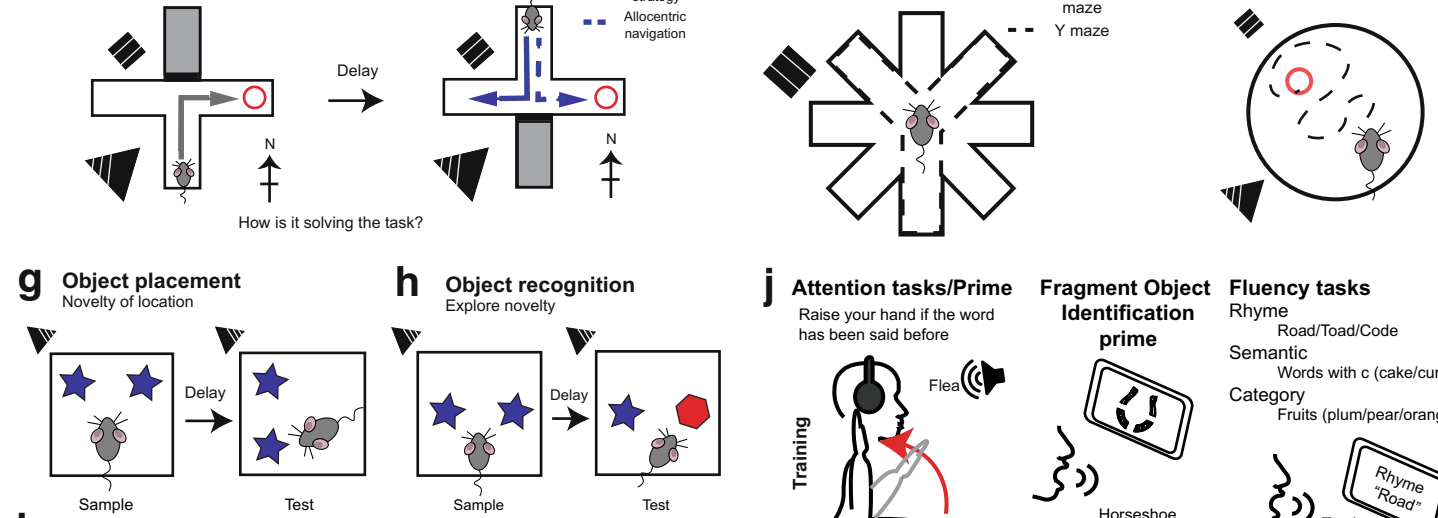

h Object recognition Explore novelty

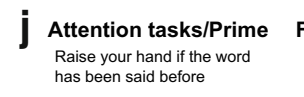

Fragment Object Fluency tasks Raise your hand if the word Identification Rhyme

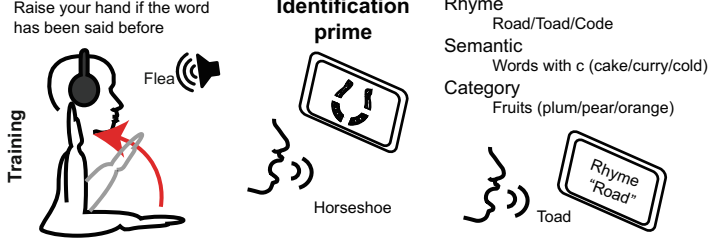

Working spatial memory

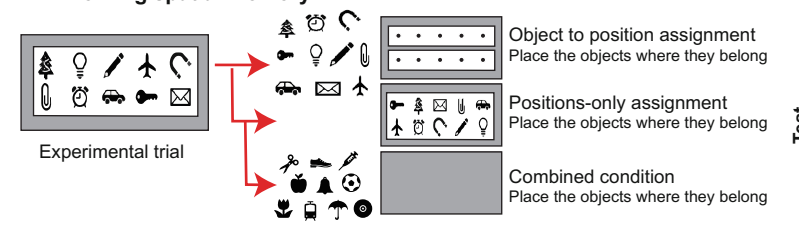

Category exemplar generation

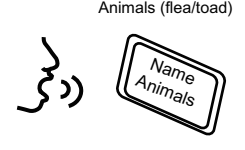

Fragmented object test Plum

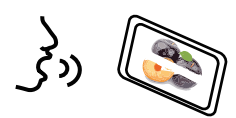

Fig. 2 Examples of tasks used for evaluating learning in humans and rodents. a Skilled reaching task; the animal has to learn to reach through a small opening, grasp a food reward and retrieve it. Tests fine motor skills. b Fear-conditioning to context; animal is placed either in the light box, or in the contiguous dark box, each paired with a specific aroma as well. Both boxes are connected but the passage is blocked once the animal moves to the contiguous context, where a foot shock is given. After a day delay, a probe is conducted, where the animal is exposed to the shock context, and the freezing time is measured, or the animal is placed in the non-shock context and the latency to enter the shock context is measured. c Example of item in an Image rotation test. A classically used task to measure spatial cognition. d Delayed matching to sample task. During several trials the rewards stays in the same arm, and the animal has to turn towards the east of the room, or turn right to get it. There are extra maze cues. During the probe, the maze is turned $180^{\circ}$, or in this case, if using a plus maze, the lower arm is cut off and the animal now starts from the north. If the animal, during the trials used an egocentric or response strategy, it would turn right to reach the goal. If, on the other hand, the animal had been using the cues to navigate towards the reward, it would have been using allocentric navigation. e Natural alternation. Either on the $\mathrm{Y}$ maze (dashed lines) or the radial arm maze (black line), animals need to remember in which arms had they been

\section{Humans}

Numerous studies [46, 47, 51] point towards men and women consistently performing differently in memory tasks according to the prevalence of verbal vs. spatial processing of information. For instance, women on average perform better in word recall and recognition, story recall, name recognition and object (including faces) recognition in complex settings. These results do not seem to be explained by verbal fluency, recently. Natural alternation between arms is counted. f Morris water maze; animal is placed in circular pool with opaque water, surrounded by extra maze cues (black square and triangle), where they have to swim to find a submerged platform (red line). The dashed line represents the swimming pattern of the rodent to reach the platform, from which distance and latency are measured. g Object placement; animal is presented in a box with two identical objects during a sample trial, with extra maze cues, and exploration time of the objects is measured. During the test trial, one of the objects is moved to a different position. $\mathbf{h}$ Object recognition. Animal is presented in a box with two identical objects during a sample trial, and exploration time of the objects is measured. During the probe, one of the objects changes identity but not position. $\mathbf{i}$ Working spatial memory test as described in Postman et al. [51]. Contains a trial stimulus showing ten objects that disappear and a test, in which objects are to be reordered as per three conditions: object to position assignment (original position marked by a dot), positions only (organize the objects in space) and the combined condition (organize the object without spatial cue). $\mathbf{j}$ Implicit memory tests, such as the fragmented object identification (middle) uses perceptual priming and category exemplar generation test (right) uses semantic categories, reported in Maki and colleagues [52]

because they remain stable even when this factor is controlled for [55]. On a different note, women perform on average worse than men in an object position memory task, even when verbal interference (repetition of meaningless syllable) was used [51]. However, the crucial element appears to be whether information can be verbally described, which gives an advantage to women (in these studies, menstrual cycle phase is typically overlooked) [55]. Also, the need to perform mental rotation of objects (see Fig. 2c) also seems to be critical, since 
women from puberty on seem to display diminished performance compared with age-matched males [56].

In contrast to navigational and spatial reasoning tasks, in verbal activities (fluency, recall of wordlists and categorial naming) women on average appear to surpass men's performance $[46,47,51,55]$. However, the origin of such differences is controversial, since performance divergence appear early in childhood (before sexual maturation) and persist after menopause and seems to be more related to divergent brain activation (more lateralized in women) than to the effect of sex hormones [46]. This verbal advantage has been connected to more precise episodic memory in women [55-57]. Thus, episodic memory and particularly memory, involving emotional arousal, seem to be favoured in women, and this advantage has been attributed to the dimorphic activation of the left amygdala in women (presumably concentrating in context details) compared with right amygdala activation in men (focusing on gist encoding) [46].

Besides physiological/structural differences between the sexes to explain the observed performance, men and women can differ in the strategy used to resolve experimental tasks (just as seen in rodents). For instance, in navigational memory, it has been reported that women rely more on egocentric cues while men incline towards allocentric references. Solving strategies in turn will affect which brain structures are involved, and in turn, different brain areas can be more or less impacted by sex hormones [55]. Concretely, as per animal evidence [58 $8^{\bullet}$, oestrogen level has been pointed out as factor modifying cognition by enhancing plasticity in key areas such as the hippocampus and prefrontal cortex [59], in female but not in males [8].

\section{Interim Summary}

In both humans and rodents, a higher performance is credited consistently to male individuals in tasks related to spatial navigation and a higher performance to females in tasks related to domains other than spatial. The factors contributing to such observed advantages are not clear and range from anatomical, and functional differences in the brain (mainly due to oestrogen level), to confounders such as stress and experimental manipulations. For instance, experimentally instilled stress before a navigational spatial task affect women but not men, independent of cycle phase [60]. In women, the presence of language and semantic processing gives another dimension to the differences in cognitive performance between sexes. As the range for variation for sex-dependent performance is wide, in the next section, we will focus on cognitive tasks that show differences in females across the menstrual phase.

\section{Influence of Menstrual Phase on Cognition}

In this section, we will summarize the influence of the menstrual phase on memory first in human subjects and then in rodents.

\section{Rodents}

Depending on the type of information needed to solve a particular task, the presence of gonadal hormones will favour or deter cognition. In tasks involving working memory, such as T maze delayed matching to sample task (Fig. 2d), which can be solved either by a place strategy (allocentric navigation/ always turning towards a location in the room) or a response strategy (egocentric navigation/always turning to the right), disambiguation of strategy use can be determined by rotating the maze $180^{\circ}$, or as shown in the figure, with a plus maze and starting from a different position within the maze. Females during proestrous are more likely to exhibit a place strategy, and during oestrous response strategies [60], both in appetitive (food reward) and aversive wet navigation tasks (submerged platform) [61]. Similarly, in ovariectomized rats treatment with oestradiol while performing a plus maze, in which place and response strategies can be evaluated, oestradiol favoured place strategies and decreased performance when response strategies were required [62].

In the $\mathrm{Y}$ maze [60] or radial arm maze (Fig. 2e), where working memory is measured by alternation, performance during oestrous was decreased compared with other stages of the cycle. Testing alternation in the T maze shows that mice during proestrous had more alternations than mice in diestrous [63]. In ovariectomized rats, performance during early acquisition in the radial arm maze was decreased compared with sham operated females [64], and treatment with oestradiol improved performance [65]. Similarly, in a water radial arm maze in which platforms were removed (which increases the working memory load as the trials advance), ovariectomized rats treated with oestradiol made fewer mistakes during the latest trials [66]. In contrast, for wet navigation tasks such as the classical Morris water maze (Fig. 2f) [67], in which performance is measured by path length and latency to find a submerged platform, rats at proestrous show decreased performance during acquisition [68] and later trials [69]. In mice, however, it seems that it is during oestrous that they have worse performance [70]. Ovariectomized rats, treated with oestradiol and oestradiol plus progesterone, decreased their performance [71]. In a variation of this navigational task, in which there is a cue hanging above the platform (a beaconing strategy) for each trial the platform location and the cue would move to a new position together, rats during proestrous exhibited better performance during acquisition than female rats in oestrous or diestrous [69]. Ovariectomized rats, treated with 
progesterone and oestradiol plus progesterone, decreased cueguided task performance [71].

In object placement (Fig. 2g) and object recognition (Fig. $2 \mathrm{~h}$ ) tasks, in which performance is evaluated as exploring time of novel versus familiar locations or objects respectively, both rats [72] and mice performing object recognition during proestrous and oestrous show better discrimination of the novel object than in diestrous or metestrous $[63,73]$, and discrimination during proestrous is better than oestrous [63]. Rats performing the object placement task showed discrimination for the novel placement only during oestrous [72]. Ovariectomized rats treated with oestradiol immediately after the sample trial showed at test trial better discrimination of the displaced or novel object. This response was dose-dependent (object placement task needed higher concentration than object recognition task, which fits into proestrous improving place and oestrous improving response strategies). Ovariectomized rats without hormone treatment showed worse performance in the object placement task than in the object recognition task, and treatment with oestradiol given 30 min before (or right after sample trial) improved discrimination in both tasks [74]. For further details in ovarian hormones regulating object recognition and object placement tasks, see [75].

\section{Humans}

It has been claimed that women's advantage in tasks in which they outperform men (verbal) intensifies during the luteal phase of the cycle when female hormones are of their highest levels. Contrarily, during women's menses, with low levels of female hormones, their performance drops in those tasks [41]. Men and women are more similar in spatial centred tasks during women's menses [76]. Classically, spatial memory and spatial cognition tasks have been used to study the change in performance for women during the different phases of their cycle. In their study, Postma and colleagues [51] showed that women displayed difference in performance only in the absolute (fine grain) positioning of objects (see an example in Fig. 2i) with respect to men and this difference was obvious when women were in their menses. The intake of oral contraceptives did not exert significant effects on position accuracy for this sample. Effects in women were not related to levels of testosterone during the menstrual phase, but more likely to the level of oestrogen.

A study by Maki and colleagues [52] showed that performance in an implicit memory task (based on Category Exemplar Generation: an example primes future word production) was better for women in the luteal phase compared with the follicular phase. On the other hand, Fragmented Object Identification (a perceptual implicit test) showed greater priming effect in the follicular phase compared with midluteal phase (see Fig. 2j). Moreover, priming effect in the perceptual modality exerted carryover effects when first encountered during the follicular phase relative to the retest during luteal phase. These results were highly correlated with estradiol level in the participants.

In terms of recollection of emotional memory (stimuli causing positive or negative arousal), Bayer and colleagues [44] found that women during their follicular phase displayed increased detail and contextual recall ( $48 \mathrm{~h}$ after encoding) of negative images compared with luteal phase and to positive and neutral images, which were not affected by menstrual phase. These differences were also correlated to decreased hippocampal (and in a portion of the accumbens) activation during the luteal phase in comparison with the follicular phase. There is also a reported differential lateralization of the amygdala during encoding of negatively arousing images, with women showing greater activation in the left amygdala and men showing the opposite pattern [77], but this asymmetry has also been reported for encoding happy faces and seems to be related to activation of other brain areas [78]. Convergently, the amygdala appeared to be activated with preference for encoding emotional memory in women during the luteal phase. Interestingly, Bayer's neuroimaging results seem to support the idea of a shift in strategy for dealing with cognitive tasks during the different phases of the menstrual cycle, with a focus on arousing emotional aspects during follicular phase (linked to anterior hippocampal activation and accumbens) and a more semantic approach in the luteal phase (with preferential activation of posterior hippocampus).

\section{Interim Summary}

Hormonal level changes during the menstrual cycle seem to have an effect on the performance of both female humans and rodents. Those changes can be related to differential activation of brain areas implicated in cognition and emotion (for instance amygdala and hippocampus), as well as use of cognitive strategies to resolve tasks during different phases of the cycle. Overall oestrogen seems to facilitate memory encoding and recall during experimental tasks, and its effects seem to be related to its action in neuronal spine density, neurogenesis, connectivity and ultimately plasticity in key brain areas as the hippocampi (for a detailed review on this topic, see the work of Hyer and colleagues [58•] and Romeo et al. [8]). A summary of these effects can be seen in Fig. 3. The effects of progesterone are less clear, and research is needed to see if has complementary or opposite effects to oestrogen $[8,44]$.

\section{Sex Differences in Sleep, Learning and Memory}

In this section, we will highlight the findings in studies that take into account both sleep and cognitive performance in 
a

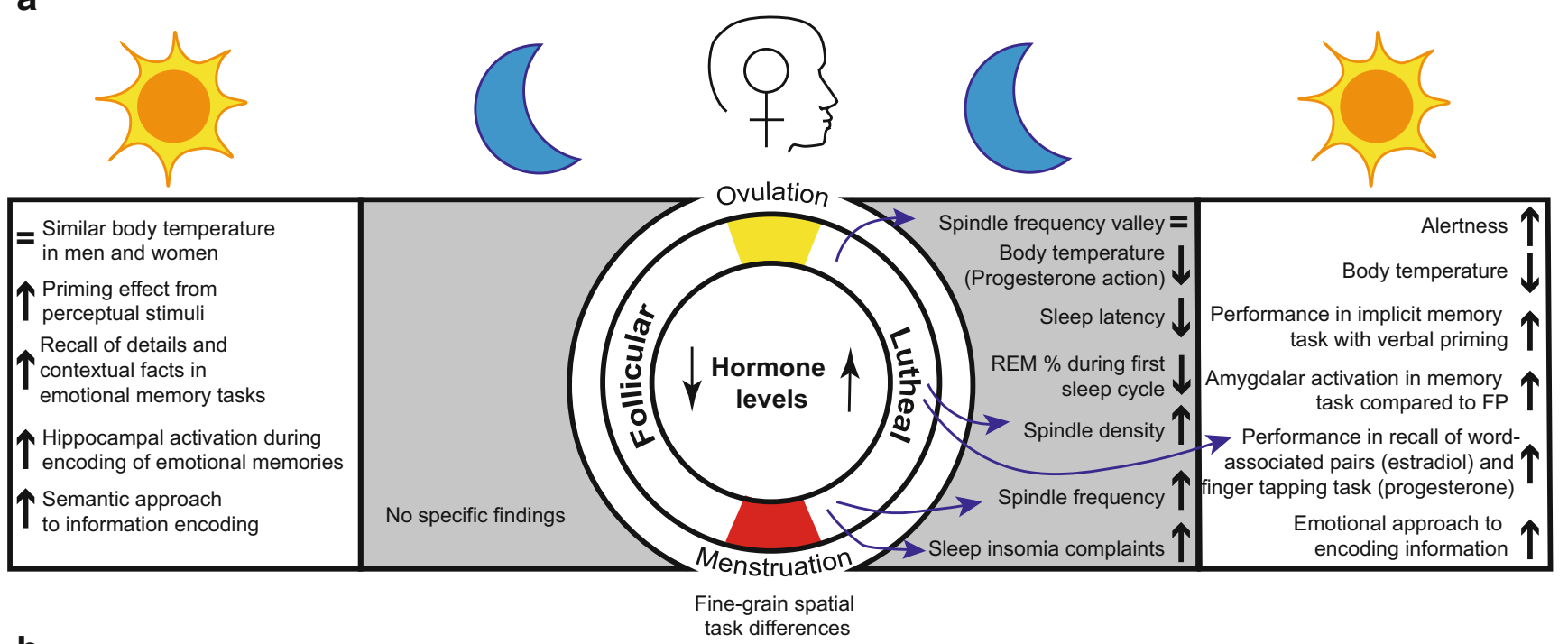

b

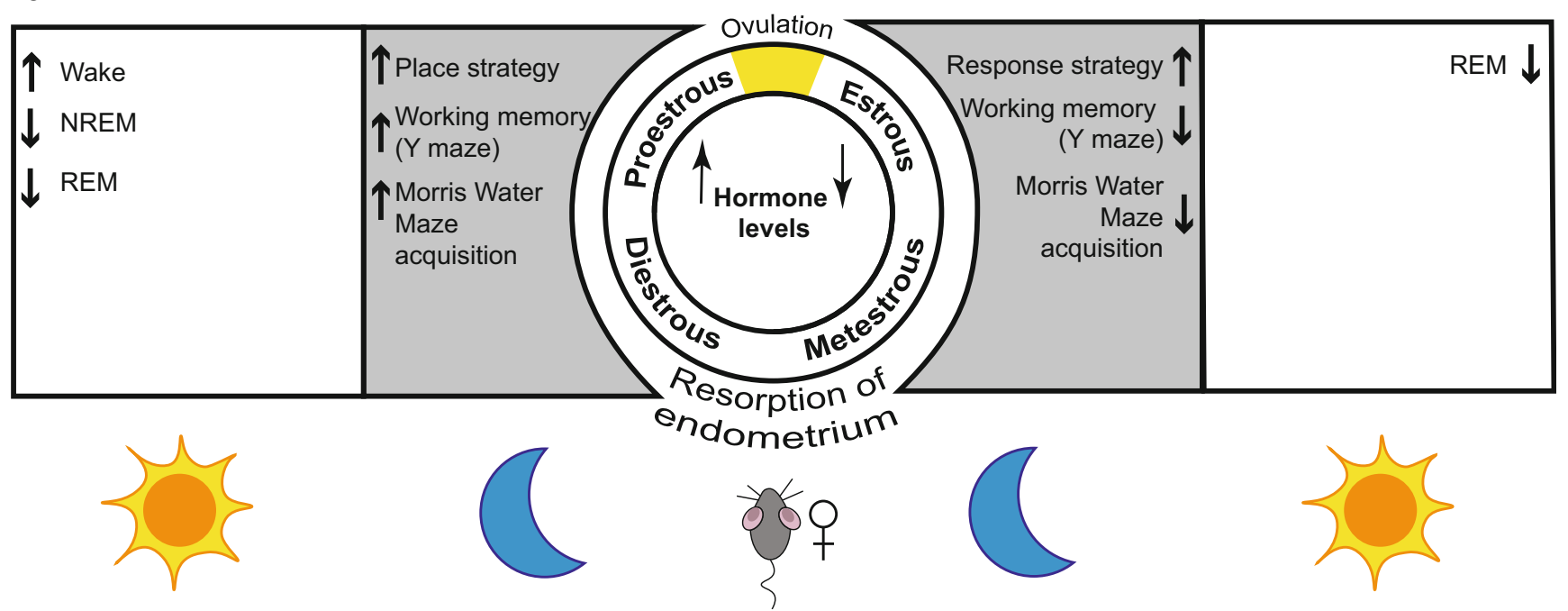

Fig. 3 Graphic summary of main findings in sleep and cognitive performance during the menstrual/oestrous cycle in women and rodents. a Main findings in humans for differences during menstrual phases in cognition and sleep. b Main findings in rodents for differences during

relationship to sex and menstrual cycle changes in human and rodent models. It is important to underscore the need for studies in this topic to better understand how these differences affect cognitive processes.

\section{Rodents}

REM sleep deprivation affected the performance in the Morris water maze of females more than of males [79], and ovariectomized rats had worse performance compared with intact females [80]. In the same page, in a fear conditioning to context task (Fig. 2b), NREM and REM sleep deprivation, before and after acquisition of the task, affected males more than females $[81 \bullet \bullet$. Rats were exposed to two contexts, one of which would be associated with a menstrual phases in cognition and sleep. Only the results for proestrous (on the left) and oestrous (on the right) are shown. The sun represents the day or light phase and the moon the night or dark phase

foot-shock. On a test $24 \mathrm{~h}$ later, the latency for the animal to enter the foot-shock context is measured. Under control conditions, males had longer latencies than females before entering the shock section, but under sleep deprivation, it was females that showed a longer latency compared with males, showing that females express a stronger fear memory than males under sleep deprivation conditions. In the object recognition task, there were no sex differences after sleep deprivation [81・•], but there was a main effect, in which the discrimination index dropped for both sexes. They also showed that after sleep deprivation, at hippocampal and cortical level, there was an elevated concentration of kynurenic acid (a metabolite of tryptophan degradation) known to affect cognition, on males but not females. This effect was lost when males were 
gonadectomized. When corticosterone levels were measured, these were higher in females than males following sleep deprivation $[81 \bullet \bullet]$.

\section{Humans}

Sex differences related to sleep-dependent performance in cognitive tasks are reflected in a small number of experimental results in humans, but there is not an integrated picture of the relationship. These findings are described next.

McDevitt and colleagues [82] reported that differences in memory consolidation after a day nap are observed for perceptual learning in a task to discriminate targets' motion direction. In this task, women showed a more generalized improvement, extending to target-direction and visual field locations not trained before sleep; while men only improved their performance for the trained motion direction. In women these findings were independent of whether the nap contained or not REM sleep. According to the authors, a candidate mechanism behind those differences could be the differential level of $\mathrm{ACh}$ hippocampal release in males and females (a fact observed in rodents but not confirmed in humans), since women in their luteal phase display an increase in this neurotransmitter that would facilitate memory performance.

Koriyama and colleagues [83] found that in relation to the circadian time for encoding aversive stimuli (violent scenes from a picture), women displayed greater accuracy in the recognition of the scenes after they have passed their habitual sleep onset periods, even when men performed better in their pre-sleep onset periods. Also, women recognized aversive scenes less accurately than neutral scenes if tested in their pre sleep-onset-periods, so women display a circadian (perhaps sleep-pressure-related) sensitivity to memory of aversive stimuli, while men display a stable pattern across time.

As mentioned in a previous section, sleep deprivation appears to have differential results in men and women. Interestingly, Binks, Waters and Hurry [84] found that sex had an effect on the IQ test measure of their sample. Sleepdeprived (36 h) females scored slightly higher than sleepdeprived males on the WAIS-R test, no pre-sleep deprivation scores were obtained from participants. Unfortunately, the authors do not discuss the implications of such finding, but it seems to contradict the claim that sleep deprivation affects women more severely in their cognitive performance [4••], as well as social jet lag impacts their academic performance more harshly [85].

A study into the differences for male and female ex users of cocaine (in recovery/abstinence) [86] allowed to see that women are not sensitive to sleep disturbances caused by abstinence (low sleep efficiency) as were men. Also importantly, women performed in a version of the motor sequence task, $24 \mathrm{~h}$ after learning it, as well as control participants and significantly better that male ex users. Female controls were not different in skill performance or sleep parameters than control males. The phase of the menstrual cycle was not controlled in this study; however, the authors speculate that oestrogens may exert protective effects on the sleep of female ex users.

\section{Interim Summary}

By observing the differential effect of sleep deprivation on memory tasks performance in males and females, the sex dimorphism consequences in sleep-dependent learning can be tackled. From these studies, it seems that under many circumstances, females perform better compared with males after sleep deprivation. As animal studies revealed, this could be related to elevated corticosteroid levels, a neuromodulator related to enhanced encoding under acute paradigms of stress. Also, in purely sleep-dependent tasks (visual memory and emotional memory) females seem to benefit more from sleep than males, these findings will be revisited in the next section.

\section{Menstrual Phase Influence on Sleep and Its Relationship with Memory}

As highlighted in the previous section, sleep-dependent learning displays differential features in female and males; in the present section, the causes attributable to variation in females due to the menstrual cycle and the implicated hormones are described.

\section{Rodents}

Very few studies take into account both sleep and learning when looking at differences between sexes and even less when looking at differences within the menstrual cycle. Cordeira et al. found that sleep deprivation negatively affected performance in Object recognition in mice during proestrous and oestrous $[73 \bullet \cdot]$.

\section{Humans}

Sleep-dependent memory consolidation is one of the fields where the influence of sleep on cognition has offered abundant evidence [87]; it is surprising that relatively few studies have explored the possible dimorphisms in this area. Below, the few studies that exist are highlighted.

Sleep-dependent memory consolidation exhibits differences in women across the menstrual cycle. Genzel and colleagues [41] demonstrated that women performed at the same level as men, during their mid-luteal phase in an associatedword-pairs and finger-tapping tasks. Overall women in their luteal phase in general, independently of the wake-sleep condition assigned to them, outperformed women in their follicular phase. The Nap-dependent performance increase, in both 
the motor and declarative tasks, was more noticeable in the luteal phase of participants compared with the follicular one. Furthermore, this nap-related-enhancement was correlated with increased spindle activity in both men and women, but specifically with progesterone level for the motor task and oestradiol level for the verbal one.

In a different type of sleep-related memory enhancement study, women did not display the effects of odour-sequence memory cueing (a well-established paradigm in the study of sleep effects on memory consolidation) during different phases of their menstrual cycle [88]. Overall, women were unaffected by the experimental manipulation, in comparison with men, for whom exposure to encoded odours during sleep resulted in performance enhancement. The fact that specific menstrual cycle effects on the observed outcomes for the female participants cannot be further explored, due to lack of experimental control of this variable, underscores the need to consider menstrual phase and the influx of external sex hormones (due to oral contraceptives) when it comes to describe the effects of sleep on memory consolidation. Since the effects of sleep can oscillate according to the momentary level of progesterone and oestrogen, the high oestrogen time window for performance enhancement due to sleep (and spindle) increase could perhaps explain the differences across samples obtained in research.

Oral contraceptives have been shown to influence cognitive task performance and memory results. For instance, Genzel and colleagues [89] showed in a sample of females that memory performance for both verbal (word-pairs) as well as motor (finger tapping) tasks was beneficially influenced by a delay in recall (whether it was filled with wake or nap time) and was comparable across active or rest weeks of oral contraceptives intake. This finding has been observed across numerous populations (for a review, see Stickgold [90] and Diekelmann, Wilhelm and Born [87]) but has been rarely studied in women using oral contraceptives. Furthermore, women taking contraceptives are rarely divided between the active and rest phase and by type of method used. The maintenance of the offline consolidation effect across contraceptive weeks can be attributed to higher levels of oestrogens (specially compared with the one of women not taking oral contraceptives) but not to an additional influence of sleep on memory consolidation.

\section{Interim Summary}

There is a lack of studies regarding the role of menstrual cycle in the relationship between sleep and its effects on learning, and concretely on memory consolidation. From the human studies, it seems plausible that different hormones affect sleep-consolidation based on the type of information being dealt with (motor or verbal, for instance). For this purpose, studies in women using oral contraceptives or suffering from irregular hormonal levels (i.e. menopause) are key to disentangle the relationship between sexual hormones and system consolidation of information in the brain.

\section{Conclusions}

The studies presented in this review underscore the fact that sex hormones, and subsequently, the menstrual cycle in females, influence sleep and cognition in a variety of ways not fully comprehended. Among those areas, we find an intersection of brain anatomical differences, activation changes in those areas, cognitive strategies to deal with a task and brain plastic changes. Overall, the results of the studies reviewed here point towards not only an effect of sex and hormones on sleep and cognition separately but also at a differential effect on the interaction of sleep and cognition. Those effects are expressed for instance as a change of strategy in spatial navigation task in both female humans and rodents (from allocentric to egocentric), in the fluctuating role of amygdala and hippocampi in encoding and consolidating stimuli in the different menstrual phases, as well as in the changes in sleep architecture and body temperature control according to hormonal level (oestrogen and progesterone). The main purpose of this review was to highlight the importance and the need to consider sex and its significances into any research endeavour. Instead of seeing sex as a confound or a variable to control for, it can be approached as an opportunity to study the role of hormones in cognition, sleep and their interplay. Since in females influence of sex hormones is more pronounced due to the cyclical variation on their level, this offers a natural milieu for the study of the impact of those substances in cognition, memory and behaviour and the influence that sleep - as a complex mechanism - exerts on them.

Innovative methods to study the relationship between sleep and cognitive performance across the phases of the menstrual cycle need to be applied, since the literature reveals that correlational and subjective measures do not seem to capture the complexity in the changes that the female brain undergoes due to the developmental and cyclical exposure to sex hormones. These sex-dependent and hormonal fluctuation variations in learning and cognitive performance can be studied as mediated by other processes, from which sleep seems to be a topic especially prolific and promising. Incorporation of female samples, accompanied by standardization of normative criteria for their parameters, into sleep research is urgently needed.

Among some of the topics to be tackled is the exact role of sex steroids in sleep regulation and its complex relationship with temperature oscillations in the mammal body. Animal studies are key to disentangle the relationship between physiological factors in sleep regulation and sleep behaviours and 
especially to separate them from cultural or psychological traits.

Based on the above, we consider that a multi-level approach to the exploration of the variables involved in the relationship between menstrual cycle, sleep and cognitive performance needs to be taken since physiological/molecular, genetic, anatomical, behavioural as wells as circadian components are simultaneously and interactively likely to produce the observed changes in cognitive performance.

Funding This work was supported by the M-GATE project, which receives funding from the European Union's Horizon 2020 research and innovation programme under the Marie Skłodowska-Curie grant agreement No 765549.

\section{Compliance with Ethical Standards}

Conflict of Interest The authors of this review certify that they have NO affiliations with or involvement in any organization or entity with any financial interest (such as honoraria; educational grants; participation in speakers' bureaus; membership, employment, consultancies, stock ownership, or other equity interest; and expert testimony or patent-licensing arrangements), or non-financial interest (such as personal or professional relationships, affiliations, knowledge, or beliefs) in the subject matter or materials discussed in this manuscript.

Human and Animal Rights and Informed Consent This article does not contain any studies with human or animal subjects performed by any of the authors.

Open Access This article is licensed under a Creative Commons Attribution 4.0 International License, which permits use, sharing, adaptation, distribution and reproduction in any medium or format, as long as you give appropriate credit to the original author(s) and the source, provide a link to the Creative Commons licence, and indicate if changes were made. The images or other third party material in this article are included in the article's Creative Commons licence, unless indicated otherwise in a credit line to the material. If material is not included in the article's Creative Commons licence and your intended use is not permitted by statutory regulation or exceeds the permitted use, you will need to obtain permission directly from the copyright holder. To view a copy of this licence, visit http://creativecommons.org/licenses/by/4.0/.

\section{References}

Papers of particular interest, published recently, have been highlighted as:

- Of importance

•. Of major importance

1. Cahill L. Why sex matters for neuroscience. Nat Rev Neurosci. 2006; 7:477-84.

2. McCarthy MM. Estradiol and the developing brain. Physiol Rev. 2008;23:1-7.

3. Mong JA, Cusmano DM, Mong JA. Sex differences in sleep : impact of biological sex and sex steroids. 2016;
4.• Hajali V, Andersen ML, Negah SS, Sheibani V. Sex differences in sleep and sleep loss-induced cognitive deficits: The influence of gonadal hormones. Horm Behav. 2019;108:50-61 This review focuses on differential effects of sleep deprivation, sleep homestasis and architecture, sex hormones in wake perfomance (in a wide range of cogntive tasks) both in rodents and humans.

5. Hadjimarkou MM, Benham R, Schwarz JM, Holder MK, Mong JA. Estradiol suppresses rapid eye movement sleep and activation of sleep-active neurons in the ventrolateral preoptic area. Eur J Neurosci. 2008;27:1780-92.

6. Manber R. Sex, steroids, and sleep: a review. Sleep. 1999;22:540-55.

7. Frick KM, Kim J, Tuscher JJ, Fortress AM. Sex steroid hormones matter for learning and memory: estrogenic regulation of hippocampal function in male and female rodents. Learn Mem. 2015;22:472-93.

8. ROMEO RD, WATERS EM, MCEWEN BS. Steroid-induced hippocampal synaptic plasticity: sex differences and similarities. Neuron Glia Biol. 2004;1:219-29.

9. Navarro-Lobato I, Genzel L. The up and down of sleep: from molecules to electrophysiology. Neurobiol Learn Mem. 2019;160:3-10.

10. Hobson JA. Sleep is of the brain, by the brain and for the brain. Nature. Nature Publishing Group; 2005. p. 1254-6.

11. Koehl M, Battle S, Meerlo P. Sex differences in sleep: the response to sleep deprivation and restraint stress in mice. Sleep. 2006;29: 1224-31.

12. Ehlen JC, Hesse S, Pinckney L, Paul KN. Sex chromosomes regulate nighttime sleep propensity during recovery from sleep loss in mice. PLoS One. 2013;8:14-9.

13. KN P, C D, FW T, AD. L. Diurnal sex differences in the sleep-wake cycle of mice are dependent on gonadal function. Sleep. Associated Professional Sleep Societies,LLC; 2006;29:1211-23.

14. Fang J, Fishbein W. Sex differences in paradoxical sleep: influences of estrus cycle and ovariectomy. Brain Res. 1996;734:275-85.

15. Deurveilher S, Rusak B, Semba K. Female reproductive hormones alter sleep architecture in ovariectomized rats (SLEEP (2011) 34, 4 (519-530)). Sleep. 2011;34:976.

16. Deurveilher S, Rusak B, Semba K. Estradiol and progesterone modulate spontaneous sleep patterns and recovery from sleep deprivation in ovariectomized rats. Sleep. 2009;32:865-77.

17. Cusmano DM, Hadjimarkou MM, Mong JA. Gonadal steroid modulation of sleep and wakefulness in male and female rats is sexually differentiated and neonatally organized by steroid exposure. Endocrinology. 2014;155:204-14.

18. Kostin A, Alam MA, Siegel JM, McGinty D, Alam MN. Sex- and age-dependent differences in sleep-wake characteristics of Fisher344 rats. Neuroscience [Internet]. IBRO. 2020;427:29-42. https:// doi.org/10.1016/j.neuroscience.2019.11.046 Features sleep changes across the oestrous cycle in young and old rats, suggesting that sleep architechture does not change much across the cycle in females, except for proestrous night.

19. Schwartz MD, Mong JA. Estradiol modulates recovery of REM sleep in a time-of-day-dependent manner. Am J Physiol - Regul Integr Comp Physiol. 2013;305:R271-80.

20. Arnold AP, Chen X. Frontiers in neuroendocrinology what does the "four core genotypes" mouse model tell us about sex differences in the brain and other tissues ? Front Neuroendocrinol [Internet]. Elsevier Inc.; 2009;30:1-9. Available from: https://doi.org/10. 1016/j.yfrne.2008.11.001, 2009.

21. Goel N, Kim H, Lao RP. Gender differences in polysomnographic sleep in young healthy sleepers. Chronobiol Int. 2005;22:905-15.

22. Voderholzer U, Al-Shajlawi A, Weske G, Feige B, Riemann D. Are there gender differences in objective and subjective sleep measures? A study of insomniacs and healthy controls. Depress Anxiety. 2003;17:162-72. 
23. Suh S, Cho N, Zhang J. Sex differences in insomnia: from epidemiology and etiology to intervention. Curr Psychiatry Rep. Current Medicine Group LLC 1; 2018;20:1-12.

24. Zhang B, Wing Y-K. Sex differences in insomnia: a meta-analysis. Sleep. 2006;29:85-93.

25. Randler C, Barrenstein S, Vollmer C, Díaz-Morales JF, Jankowski KS. Women would like their partners to be more synchronized with them in their sleep-wake rhythm. Span J Psychol Cambridge University Press; 2014;17.

26. Santhi N, Lazar AS, McCabe PJ, Lo JC, Groeger JA, Dijk DJ. Sex differences in the circadian regulation of sleep and waking cognition in humans. Proc Natl Acad Sci U S A National Academy of Sciences. 2016;113:E2730-9.

27. Carrier J, Land S, Buysse DJ, Kupfer DJ, Monk TH. The effects of age and gender on sleep EEG power spectral density in the middle years of life (ages 20-60 years old). Psychophysiology WileyBlackwell. 2001;38:232-42.

28. Fernandez LMJ, Lüthi A. Sleep Spindles: Mechanisms and Functions. Physiol Rev. NLM (Medline); 2020;100:805-68.

29. Ujma PP, Konrad BN, Genzel L, Bleifuss A, Simor P, Pótári A, et al. Sleep spindles and intelligence: evidence for a sexual dimorphism. J Neurosci. 2014;34:16358-68.

30. Driver HS, Dijk D-J, Werth E, Biedermann K, Borbély AA. Sleep and the sleep electroencephalogram across the menstrual cycle in young healthy women*. J Clin Endocrinol Metab. 1996:729-35.

31. Plante DT, Goldstein MR, Landsness EC, Peterson MJ, Riedner BA, Ferrarelli F, et al. Topographic and sex-related differences in sleep spindles in major depressive disorder: a high-density EEG investigation. J Affect Disord NIH Public Access. 2013;146:120-5.

32. Huupponen E, Sari-Leena H, Varri A, Hasan J, Lehtokangas M, Saarinen J. A study on gender and age differences in sleep spindles. Neuropsychology. 2002;45:99-105.

33.• Sattari N, McDevitt EA, Panas D, Niknazar M, Ahmadi M, Naji M, et al. The effect of sex and menstrual phase on memory formation during a nap. Neurobiol Learn Mem. 2017;145:119-28. https://doi. org/10.1016/j.nlm.2017.09.007 This article connects sex differeces, menstrual cycle phase with perfomance in a facename association after a nap, and additionally shows that oscillatory events change their relative importance in sleepconsolidation with menstrual phase.

34. Becker JB, Arnold AP, Berkley KJ, Blaustein JD, Eckel LA, Hampson E, et al. Strategies and methods for research on sex differences in brain and behavior. Endocrinology. 2005;146:1650-73.

35. Koehl M, Battle SE, Turek FW. Sleep in female mice: a strain comparison across the estrous cycle 2003;26.

36. Driver HS, Werth E, Dijk DJ, Borbély AA. The menstrual cycle effects on sleep. Sleep Med Clin. 2008;3:1-11.

37. McEwen BS, Milner TA. Understanding the broad influence of sex hormones and sex differences in the brain. J Neurosci Res. John Wiley and Sons Inc.; 2017;95:24-39.

38. Romans SE, Kreindler D, Einstein G, Laredo S, Petrovic MJ, Stanley J. Sleep quality and the menstrual cycle. Sleep Med. 2015;16:489-95.

39. Wright KP, Badia P. Effects of menstrual cycle phase and oral contraceptives on alertness, cognitive performance, and circadian rhythms during sleep deprivation. Behav Brain Res Elsevier. 1999;103:185-94.

40. Baker FC, Driver HS. Circadian rhythms, sleep, and the menstrual cycle. Sleep Med. 2007;8:613-22.

41. Genzel L, Kiefer T, Renner L, Wehrle R, Kluge M, Grözinger M, et al. Sex and modulatory menstrual cycle effects on sleep related memory consolidation. Psychoneuroendocrinology. 2012;37:987-98.

42. Y I, CP P, Shirakawa S, Kakuma T AK, A U, K S, H F, et al. Sleep spindle frequency changes during the menstrual cycle. J Sleep Res. John Wiley \& Sons, Ltd; 1994;3:26-9.
43. Plante DT, Goldstein MR. Medroxyprogesterone acetate is associated with increased sleep spindles during non-rapid eye movement sleep in women referred for polysomnography. Psychoneuroendocrinology. NIH Public Access. 2013;38:3160-6.

44. Bayer J, Schultz H, Gamer M, Sommer T. Menstrual-cycle dependent fluctuations in ovarian hormones affect emotional memory. Neurobiol Learn Mem. 2014;110:55-63.

45. Spencer RMC, Walker MP, Stickgold R. Sleep and memory consolidation. Sleep Disord Med Basic Sci Tech Considerations Clin Asp Fourth Ed. Springer New York; 2017;205-23.

46. Andreano JM, Cahill L. Sex influences on the neurobiology of learning and memory. Learn Mem. 2009;16:248-66.

47. Mizuno K, Giese KP. Towards a molecular understanding of sex differences in memory formation. Trends Neurosci. Elsevier Current Trends. 2010;33:285-91.

48. Kanit L, Taskiran D, Yilmaz ÖA, Balkan B, Demirgören S, Furedy JJ, et al. Sexually dimorphic cognitive style in rats emerges after puberty. Brain Res Bull. 2000;52:243-8.

49. Jonasson Z. Meta-analysis of sex differences in rodent models of learning and memory: a review of behavioral and biological data. Neurosci Biobehav Rev. 2005;28:811-25.

50. Gresack JE, Frick KM. Male mice exhibit better spatial working and reference memory than females in a water-escape radial arm maze task. Brain Res. 2003;982:98-107.

51. Postma A, Winkel J, Tuiten A, van Honk J. Sex differences and menstrual cycle effects in human spatial memory. Psychoneuroendocrinology. 1999;24:175-92.

52. Maki PM, Rich JB, Rosenbaum S. Implicit memory varies across the menstrual cycle: estrogen effects in young women. Neuropsychologia. 2002;40:518-29.

53. Keeley RJ, Bye C, Trow J, McDonald RJ. Strain and sex differences in brain and behaviour of adult rats: learning and memory, anxiety and volumetric estimates. Behav Brain Res [Internet]. Elsevier B.V.; 2015;288:118-31. Available from: https://doi.org/ 10.1016/j.bbr.2014.10.039, 2015.

54. Roof RL, Stein DG. Gender differences in Morris water maze performance depend on task parameters. Physiol Behav. 1999;68:81-6.

55. Lewin C, Wolgers G, Herlitz A. Sex differences favoring women in verbal but not in visuospatial episodic memory - PubMed. Neuropsychology. 2001;15:165-73.

56. Levine SC, Foley A, Lourenco S, Ehrlich S, Ratliff K. Sex differences in spatial cognition: advancing the conversation. WIREs Cogn Sci. 2016;7:127-55.

57. Catharina L, Wolgers G, Herlitz A. Sex differences in episodic memory: the impact of verbal and visuospatial ability The Emerging Elderly Population in Bangladesh View project Aging along the hippocampal axis View project. Neuropsychology. 1999;15:165-73.

58. Hyer MM, Phillips LL, Neigh GN. Sex differences in synaptic plasticity: hormones and beyond. Front Mol Neurosci. Frontiers Media S.A. 2018;11:266 Interesting review on the complex aspects of sex hormones and mechanisms of synaptic plasticity. Of special importance is the hippocampal differences between female and males.

59. Diaz BR. Estrogen-induced plasticity from cells to circuits: predictions for cognitive function. Trends Pharmacol Sci. 2009;30:212-22.

60. Korol DL, Malin EL, Borden KA, Busby RA, Couper-Leo J. Shifts in preferred learning strategy across the estrous cycle in female rats. Horm Behav. 2004;45:330-8.

61. Hawley WR, Grissom EM, Barratt HE, Conrad TS, Dohanich GP. The effects of biological sex and gonadal hormones on learning strategy in adult rats. Physiol Behav [Internet]. Elsevier Inc.; 2012;105:1014-20. Available from: https://doi.org/10.1016/j. physbeh.2011.11.021, 2012. 
62. Korol DL, Kolo LL. Estrogen-induced changes in place and response learning in young adult female rats. Behav Neurosci. 2002;116:411-20.

63. Walf AA, Koonce C, Manley K, Frye CA. Proestrous compared to diestrous wildtype, but not estrogen receptor beta knockout, mice have better performance in the spontaneous alternation and object recognition tasks and reduced anxiety-like behavior in the elevated plus and mirror maze. Behav Brain Res. 2009;196:254-60.

64. Daniel JM, Roberts SL, Dohanich GP. Effects of ovarian hormones and environment on radial maze and water maze performance of female rats working memory reference memory learning radial maze water maze ovarian hormones estradiol estrogen progesterone complex environment. Physiol Behav. 1999;66:11-20.

65. Daniel JM, Fader AJ, Spencer A, Dohanic G. Estrogen enhances performance of female rats during acquisition of a radial arm maze. Horm Behav JID - 0217764. 1997;32:217-25.

66. Bimonte HA, Denenberg VH. Estradiol facilitates performance as working memory load increases. Psychoneuroendocrinology. 1999;24:161-73.

67. Morris R. Spatial localization does not require the presence of local cues. Learn Motiv. 1981;12:239-60.

68. Korol DL. Role of estrogen in balancing contributions from multiple memory systems. Neurobiol Learn Mem. 2004;82:309-23.

69. Warren SG, Juraska JM. Spatial and nonspatial learning across the rat estrous cycle. Behav Neurosci. 1997;111:259-66.

70. Frick KM, Berger-Sweeney J. Spatial reference memory and neocortical neurochemistry vary with estrous cycle in c57BL/6 mice. 2001.

71. Chesler EJ, Juraska JM. Acute administration of estrogen and progesterone impairs the acquisition of the spatial Morris water maze in ovariectomized rats. Horm Behav. 2000;38:234-42.

72. Sutcliffe JS, Marshall KM, Neill JC. Influence of gender on working and spatial memory in the novel object recognition task in the rat. Behav Brain Res. 2007;177:117-25.

73.• Cordeira J, Kolluru SS, Rosenblatt H, Kry J, Strecker RE, McCarley RW. Learning and memory are impaired in the object recognition task during metestrus/diestrus and after sleep deprivation. Behav Brain Res. 2018;339:124-9. https://doi.org/10.1016/j.bbr.2017.11. 033 Takes into consideration both oestrous cycle and sleep on cognition.

74. Inagaki $\mathrm{T}$, Gautreaux $\mathrm{C}$, Luine $\mathrm{V}$. Acute estrogen treatment facilitates recognition memory consolidation and alters monoamine levels in memory-related brain areas. Horm Behav [Internet]. Elsevier Inc.; 2010;58:415-26. Available from: https://doi.org/10. 1016/j.yhbeh.2010.05.013, 2010.

75. Tuscher JJ, Fortress AM, Kim J, Frick KM. Regulation of object recognition and object placement by ovarian sex steroid hormones. Behav Brain Res [Internet]. Elsevier B.V.; 2015;285:140-57. Available from: https://doi.org/10.1016/j.bbr.2014.08.001, 2015.

76. Barel E, Maayan Krispil, Inbar Yaari. Cognitive performance across the menstrual cycle. J Psychol Cogn. Allied Academies; $2019 ; 4$.
77. Cahill L, Uncapher M, Kilpatrick L, Alkire MT, Turner J. Sexrelated hemispheric lateralization of amygdala function in emotionally influenced memory: an fMRI investigation. Learn Mem Learn Mem. 2004;11:261-6.

78. Kilpatrick LA, Zald DH, Pardo JV, Cahill LF. Sex-related differences in amygdala functional connectivity during resting conditions. Neuroimage. 2006;30:452-61.

79. Hajali V, Sheibani V, Esmaeili-Mahani S, Shabani M. Female rats are more susceptible to the deleterious effects of paradoxical sleep deprivation on cognitive performance. Behav Brain Res [Internet]. Elsevier B.V.; 2012;228:311-8. Available from: https://doi.org/10. 1016/j.bbr.2011.12.008, 2012.

80. Esmaeilpour K, Sheibani V, Saadati H. Caffeine improved spatial learning and memory deficit in sleep deprived female rat. Physiol Pharmacol. 2015;19:121-9.

81.• Baratta AM, Buck SA, Buchla AD, Fabian CB, Chen S, Mong JA, et al. Sex differences in hippocampal memory and kynurenic acid formation following acute sleep deprivation in rats. Sci Rep. 2018;8:1-11. https://doi.org/10.1038/s41598-018-25288-w Sex differences under sleep deprivation and the impact on memory.

82. McDevitt EA, Rokem A, Silver MA, Mednick SC. Sex differences in sleep-dependent perceptual learning. Vision Res. 2014;99:172-9.

83. Kuriyama K, Mishima K, Soshi T, Honma M, Kim Y. Effects of sex differences and regulation of the sleep-wake cycle on aversive memory encoding. Neurosci Res. 2011;70:104-10.

84. Binks PG, Waters WF, Hurry M. Short-term total sleep deprivations does not selectively impair higher cortical functioning. Sleep. 1999;22:328-34.

85. Díaz-Morales JF, Escribano C. Social jetlag, academic achievement and cognitive performance: understanding gender/sex differences. Chronobiol Int. 2015;32:822-31.

86. Morgan PT, Paliwal P, Malison RT, Sinha R. Sex differences in sleep and sleep-dependent learning in abstinent cocaine users. Pharmacol Biochem Behav. NIH Public Access. 2009;93:54-8.

87. Diekelmann S, Wilhelm I, Born J. The whats and whens of sleepdependent memory consolidation. Sleep Med Rev. W.B. Saunders. 2009;13:309-21.

88. Diekelmann S, Born J, Rasch B. Increasing explicit sequence knowledge by odor cueing during sleep in men but not women. Front Behav Neurosci. 2016;10:1-11.

89. Genzel L, Bäurle A, Potyka A, Wehrle R, Adamczyk M, Friess E, et al. Diminished nap effects on memory consolidation are seen under oral contraceptive use. Neuropsychobiology. 2015;70:253-61.

90. Stickgold R. INSIGHT REVIEW Sleep-dependent memory consolidation. Nature. 2005;437:1207-396.

Publisher's Note Springer Nature remains neutral with regard to jurisdictional claims in published maps and institutional affiliations. 